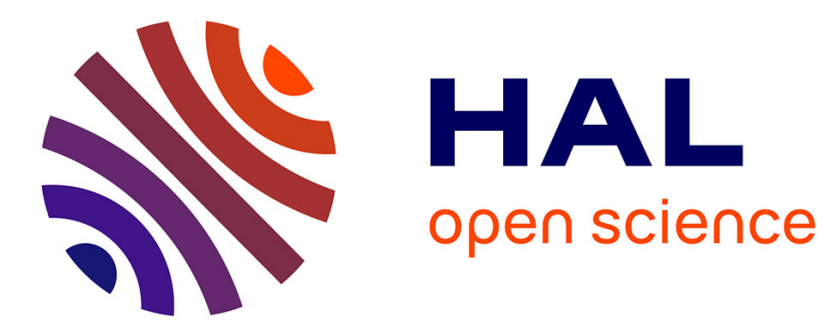

\title{
Entropic Estimation of Noise for Medical Volume Restoration
}

\author{
Marc Liévin, Franck Luthon, Erwin Keeve
}

\section{To cite this version:}

Marc Liévin, Franck Luthon, Erwin Keeve. Entropic Estimation of Noise for Medical Volume Restoration. 16th International Conference on Pattern Recognition, ICPR 2002, Aug 2002, Quebec, Canada. pp.871-874, 10.1109/ICPR.2002.1048166 . hal-01912840

\section{HAL Id: hal-01912840 \\ https://hal-univ-pau.archives-ouvertes.fr/hal-01912840}

Submitted on 1 Sep 2019

HAL is a multi-disciplinary open access archive for the deposit and dissemination of scientific research documents, whether they are published or not. The documents may come from teaching and research institutions in France or abroad, or from public or private research centers.
L'archive ouverte pluridisciplinaire HAL, est destinée au dépôt et à la diffusion de documents scientifiques de niveau recherche, publiés ou non, émanant des établissements d'enseignement et de recherche français ou étrangers, des laboratoires publics ou privés. 


\section{Entropic Estimation of Noise for Medical Volume Restoration}

\author{
Marc Liévin \\ Research center caesar \\ 53111 Bonn, Germany \\ lievin@caesar.de
}

\author{
Franck Luthon \\ Univ. of Pau and Adour \\ 64115 Bayonne, France \\ Franck.Luthon@univ-pau.fr
}

\author{
Erwin Keeve \\ Research center caesar \\ 53111 Bonn, Germany \\ keeve@caesar.de
}

\begin{abstract}
This paper presents an unsupervised approach for medical volume restoration. To cope with various scanning modalities and strongly corrupted data, an original information tool is introduced: the entropic deviation. To validate the robustness of this estimation, a non-linear restoration filter based on Markov random fields is proposed. No parameter tuning is required from the user thanks to the adaptive value of the entropy power. Finally, the good quality of the filtered volumes are promising for any clustering application aiming at anatomical structures extraction in medical volume datasets.
\end{abstract}

\section{Introduction}

Image restoration techniques are widely used in the field of medical imaging, where human interpretation is linked with interactivity for challenging anatomical segmentation. Medical images are actually strongly deteriorated by noise mainly due to data acquisition systems. While signal-tonoise ratio (SNR) is improving in such scanning systems, higher resolutions are still required in surgical applications. As a main consequence, the noise level remains high for an elementary volumetric data, i.e. voxel.

Several methods coexist in the medical literature, each satisfying a subset of requirements. Our aim is to propose a restoration technique that is meant to be used by subsequent algorithms for medical image processing, namely connectivity filters. Therefore, the restoration should be unsupervised to propose interactivity for non-expert users while keeping relevant anatomical information.

Advanced medical restoration techniques can be categorized in two major groups. The first one concerns optimal filters, adapting to local and spatial information in images. For instance, the Wiener filter can be combined with a visibility function depending on the magnitude of the image gradient vector [1]. Recently, the adaptive theory has been extended to 3-D space and collections of filters [11]. Non- linear wavelet filtering has proved to be useful for specific applications (e.g. mammography enhancement) [3]. Unfortunately, the shape of the filters depends on the nature of the corrupting noise. To make a compromise between stability and smoothness, this parameter is generally manually tuned. The second category proposes nonlinear statistical estimation [6]. Restoration may be efficient by using Markov random fields but the sensitivity to structures distributions is a major limitation [7]. Moreover, such Bayesian approaches are very dependant on the normalization level parameters. Simulated annealing has been applied on the parameters estimation to reduce its sensitivity [2]. To our knowledge, few methods for estimating the amount of noise in images have been proposed [9]. Prior knowledges and beforehand determined parameters are the common solution chosen for medical image restoration.

Two methods based on absolute difference are tested in this paper. Image difference can be considered as classic method in image registration or noise estimation [9]. Two measurements are then applied on these observations: the empirical standard deviation estimation and an entropic deviation computation. On the opposite to generic techniques that use entropy as a fitting criterion, this measure is used to estimate the power of noise. Next, a dedicated Markov random fields restoration process is presented, taking account of the entropy and volumetric constraint potentials. Finally, the robustness of the results is evaluated on synthetic data and real datasets representing several medical modalities.

\section{Noise Deviation Estimation}

\subsection{Noise Estimation}

We model the medical volume data $V$ as a collection of slices $S_{i}$, corrupted by an independant additive Gaussian noise $G(0, \sigma)$. This rough assumption respects however the classic model of image backprojection acquisition and often corresponds to a good approximation in actual cases [8]. Tomographic images are actually corrupted by a Rician 
noise, result of the inverse Radon transform, that can be approximated by Gaussian distributions in noisy environment.

The two estimations tested are defined as:

1. The difference between the slice $S_{i}$ and its Gaussian filtered image $\nabla S_{i}, s f_{i}=\left|S_{i}-\nabla S_{i}\right|$. This observation is therefore directly related to the noise and the spatial edges of the slice. The variance is generally under-estimated.

2. The slice difference $s d_{i}=\left|S_{i+1}-S_{i}\right|$, that contains information on the noise $G(0, \sqrt{2} \sigma)$ and on the vertical gradient of anatomical objects of the volume. The variance is generally over-estimated.

The empirical standard deviation std is a generic measure of the power of Gaussian distribution $\left\{x_{i}\right\}$ but sensitive to outliers:

$$
s t d^{2}=\frac{1}{N-1} \sum_{i=1}^{N}\left(x_{i}-\bar{x}\right)^{2} .
$$

The following paragraph introduces a new estimation of the noise deviation.

\subsection{Entropic Standard Deviation}

As defined by Shannon in [10], the entropy $E(X)$ of a discrete source $X$ of observations taking values in the set $\{i, i \in[0 \cdots M-1]\}$ is a measure of its mean information:

$$
E(X)=-\sum_{i=0}^{M-1} p_{i} \log \left(p_{i}\right)
$$

where $p_{i}$ is the probability of the event $i$.

For an arbitrary source with a given entropy $E(X)$, Shannon also defines the notion of entropy power $N(X)$, which represents the power of the white noise equivalent to source $X$, in the sense it has the same entropy and is limited to the same band:

$$
N(X)=\frac{1}{2 \pi e} e^{2 \cdot E(X)} .
$$

The two important properties of the entropy power are:

- The entropy power of the sum of two signals is lowerbounded by the sum of their respective entropy powers, and upper-bounded by the sum of their actual average powers.

- The Gaussian source has the maximum entropy for a given power, verifying $E(G)=\log (\sigma \sqrt{2 \pi e})$. Hence, its entropy power equals its power: $N(G)=\sigma^{2}$. As a consequence, the entropy power of any source is always less than or equal to its actual power.
According to our additive model, the noise estimation $\mathbf{n}$ (i.e. $s d$ or $s f$ ) is the sum of a relevant signal $X$ plus an additive Gaussian noise $G(0, \sigma)$. The first property yields the following inequality, where $P_{X}$ is the average power of the gradient signal:

$$
N(X)+N(G) \leq N(\mathbf{n}) \leq P_{X}+\sigma^{2} .
$$

Applying the second property, this inequality becomes:

$$
N(X) \leq N(\mathbf{n})-\sigma^{2} \leq P_{X} .
$$

White Gaussian noise has the peculiar property that it can absorb any other signal which may be added to it, provided the signal power is small, in a certain sense, compared to noise [10]. The resultant entropy power is therefore approximately equal to the sum of the white noise power and the signal power. Considering that relevant changes between slices are localized on the object frontiers in the image, whereas noise is spread over the entire frame, the average power $P_{X}$ of the difference source is small compared to the noise power $\sigma^{2}$ (i.e., the average SNR over the difference between slices is small, although the local SNR may be high). Therefore, we have:

$$
N(\mathbf{n}) \underset{P_{X} \ll \sigma^{2}}{\sim} P_{X}+\sigma^{2}
$$

In summary, the entropy power is a measure that provides a means to evaluate the power of the noise in the slices. We call entropic standard deviation $\kappa_{i}$ of the noise observation $\mathbf{n}_{i}$ :

$$
\kappa_{i}=\sqrt{N\left(\mathbf{n}_{i}\right)}=\frac{1}{\sqrt{2 \pi e}} e^{E\left(\mathbf{n}_{i}\right)} .
$$

\subsection{Noise Deviation Estimation in Medical Tomo- graphic Images}

Four tomographic volumes in magnetic resonance (MR) and X-ray computed tomography (CT) modalities have been evaluated. These medical volumes include 2 different modalities and 3 anatomical structures, i.e. head, knee and pelvis. One synthetic data have been tested respecting medical data level properties ${ }^{1}$. Table 1 details the cases studied in this paper.

Table 2 gives the results of the noise deviation estimation methods $(s t d, \kappa)$ when applied on the image difference observations $(s d, s f)$. Manual estimation has been made by manual determination of a subvolume of interest with no anatomical structures and homogeneous background, the deviation corresponds to the geometrical mean of the proposed methods. The synthetic data (case 5) reveals that the

\footnotetext{
${ }^{1}$ The synthetic data coded on 12 bits consists in a sphere of value 2048 on a background of 256 , corrupted by an additive noise $G\left(0,50^{2}\right)$.
} 


\begin{tabular}{|c||c|c|c|}
\hline Case & Modality & Anatomy & Size (x,y,slices) \\
\hline 1 & CT & Pelvis & $512 \times 512 \times 46$ \\
\hline 2 & CT & Head & $320 \times 372 \times 92$ \\
\hline 3 & MR & Knee & $512 \times 512 \times 121$ \\
\hline 4 & MR & Head & $256 \times 256 \times 136$ \\
\hline 5 & Synthetic & Sphere & $256 \times 256 \times 128$ \\
\hline
\end{tabular}

Table 1. Cases studied for noise estimation.

\begin{tabular}{|c||c|c||c|c||c|}
\hline \multicolumn{1}{|c||}{ Case } & \multicolumn{2}{c|}{$s d$} & \multicolumn{2}{c|}{$s f$} & \multirow{2}{*}{ Manual } \\
\cline { 2 - 5 } & $s t d$ & $\kappa$ & $s t d$ & $\kappa$ & \\
\hline 1 & 56.8 & $\mathbf{1 9 . 1}$ & 21.5 & 9.56 & 16.3 \\
& $(.15)$ & $(. \mathbf{0 8})$ & $(.13)$ & $(.10)$ & \\
\hline 2 & 76.6 & $\mathbf{1 0 . 8}$ & 23.5 & 6.54 & 9.2 \\
& $(.30)$ & $(.31)$ & $(.23)$ & $(.27)$ & \\
\hline 3 & 182 & $\mathbf{1 5 6}$ & 93 & 88 & 130 \\
& $(.10)$ & $(.07)$ & $(.08)$ & $(.07)$ & \\
\hline 4 & 21.5 & 12.4 & 11.2 & $\mathbf{8 . 3 1}$ & 9.6 \\
& $(.13)$ & $(.14)$ & $(.23)$ & $\mathbf{( . 2 1})$ & \\
\hline 5 & 162 & 54.6 & 86.5 & $\mathbf{4 6 . 9}$ & 50.0 \\
& $(.31)$ & $(.04)$ & $(.12)$ & $\mathbf{( . 0 4})$ & \\
\hline
\end{tabular}

Table 2. Noise deviation estimation, where empirical deviation, std and entropic estimation, $\kappa$ are applied on slice difference, $s d$ and slice difference with its filtered value, $s f$. In bold, the closest value to manual measurement. Between braces, the "stability" criteria.

empirical deviation is very sensitive to gradients whereas the entropic measure stays close to the actual noise level, $\sigma=50$. CT datasets (cases 1,2) are known to be much less corrupted by noise than MR datasets (cases 3). Only the entropic estimation respects this difference in a ratio of 10 whereas the empirical deviation is less convincing. These results show that the entropic deviation when combined with slices difference is an accurate and reliable measurement of the noise level.

Constancy of the estimation along the slices is mandatory for a reliable volume restoration. Table 2 details between braces values of the variance in a $\mathrm{z}$-score form, i.e. the distribution is normalized by the ratio between the variance and the mean value. This criteria reveals the "stability" of the methods along the volume slices. The empirical estimation appears to have the lowest stability (higher values), e.g. the difference with the entropic stability measurement is at minimum $20 \%$ and can reached $50 \%$. The entropic deviation is therefore a better criteria when no manual tuning is allowed: the estimate is little biased by anatomy variations and its repetability can be guaranteed.

\section{Volumetric Restoration}

\subsection{Volumetric Markov random fields}

To confirm the quality and the stability of the entropic estimation, we integrate this measure in a restoration framework based on volumetric Markov random fields (MRF). The label field is supposed to follow the Gibbs property related to a neighborhood structure, i.e. the label of a voxel depends only on the labels of its neighbors and associated probabilities are modeled as Gibbs functions. In Bayesian context, Maximizing the A Posteriori probability (MAP criterion) of the label field is equivalent to minimize a global energy function [4]:

$$
U=U_{l}+\alpha \cdot U_{p}
$$

where $U_{l}$ represents the likelihood energy expressing the link between labels and observations, $U_{p}$ defines the prior energy corresponding to spatial constraints, $\alpha$ is a weighting coefficient between the two energies.

In the following, $v(x, y, z)$ is a voxel of the volume $V$, $n$ denotes a neighbor of $v$ belonging to its neigborhood $\eta$. The restoration purpose is to find the closest label $l_{v}$ corresponding to the observation $o_{v}$ of the original data. As in the rest of the paper, we consider an additive and centered Gaussian noise with a standard deviation $\sigma$.

The two energies are expressed as:

$$
\begin{aligned}
U_{l}(V) & =\sum_{v \in V}\left[\frac{\left(o_{v}-l_{v}\right)^{2}}{2 \sigma^{2}}\right] \\
U_{p}(V) & =\sum_{v \in V}\left[\sum_{n \in \eta(v)} V_{p}(v, n)\right]
\end{aligned}
$$

where $V_{p}$ defines spatial prior constraints.

We propose to construct the metric prior functions $V_{p}$ as the inverse of the Euclidian distance between two neighbors. These potentials constraint the field towards homogeneity respecting the data spacing defined in millimeters $\left(\delta_{x}, \delta_{y}, \delta_{z}\right)$ :

$$
V_{p}(v, n)= \begin{cases}\frac{-\delta_{V}}{\|\vec{v}-\vec{n}\|} & \text { if } l_{v}=l_{n} \\ \frac{+\delta_{V}}{\|\vec{v}-\vec{n}\|} & \text { otherwise. }\end{cases}
$$

where $\|\vec{v}-\vec{n}\|$ denotes the Euclidian distance between the vectors $\vec{v}$ and $\vec{n}, \delta_{V}=\sqrt{\delta_{x}^{2}+\delta_{y}^{2}+\delta_{z}^{2}}$ is the average volumetric spacing of the data. Our neighborhood structure $\eta(v)$ contains 26 neighbors in a 3-D metric space.

The iterative deterministic algorithm ICM (Iterated Conditional Modes) is implemented to compute the minimum energy at each voxel. The entropic deviation $\kappa$ provides the estimation of the noise variance.

The local energy $u_{i, v}$ in the slice $i$ at the voxel $v$ can be expressed as the following regularization function:

$$
u_{i, v}=\left(o_{v}-l_{v}\right)^{2}+2 . \alpha \cdot \kappa_{i}^{2} \cdot \sum_{n \in \eta(v)} V_{p}(v, n)
$$


The initial label configuration is the data itself. The normalization coefficient depends only on the range of the potential values, i.e. the size of the neighborhood. Typical values of $\alpha$ are 1 and 0.2 , respectively in a 6 and 26 neighbors system. Less than 10 iterations on the field are enough to respect the stopping criterion for convergence $\Delta U / U<0.05 \%$.

\subsection{Results}

Figure 1 compares results of 3-D filtering techniques when applied on a MR volume of the brain. The original slice reveals two distinguable but noisy areas of the brain: the white matter in light grey and the grey matter in dark grey. Whereas the median filter smoothes the boundaries, the anisotropic diffusion filter works well by keeping the initial edges [5] ${ }^{2}$. Nonetheless, the MRF volume produces sharp edges and homogeneizes regions without user tuning. The distinction between anatomical areas is increased and proposes good preprocessing for any following segmentation process. The stability of the estimate provides also few variations in the quality of the filtering: the sharpness is constant along the slices independantly of the anatomy.

\section{Discussion}

In conclusion, the entropic deviation estimation has proved to be accurate, reliable and robust when applied on medical volumes independantly of the modality or the anatomy studied. When combined with MRF restoration, this method proposes an unsupervised way to remove the noise and sharpen the edges of anatomical structures. Contrary to empirical variance approaches, the quality of the results is constant along the slices.

The relevance of the entropic deviation is also under study for gradient and motion estimation. Other filters should gain from this noise estimation, like anisotropic diffusion or wavelet coefficients reduction.

The complete restoration pipeline is currently integrated in an interactive segmentation framework for maxillofacial surgery planning. First results are promising thanks to the robustness of the method.

\section{References}

[1] J. Abramatic and L. Silverman. Nonlinear restoration of noisy images. IEEE Trans. PAMI, 4(2):141-149, 1982.

[2] R. Aykroyd. Bayesian estimation for homogeneous and inhomogeneous Gaussian random fields. IEEE Trans. PAMI, 20(5):533-539, 1998.

\footnotetext{
${ }^{2}$ The threshold on the gradient magnitude may be defined as the entropic estimation of the noise.
}

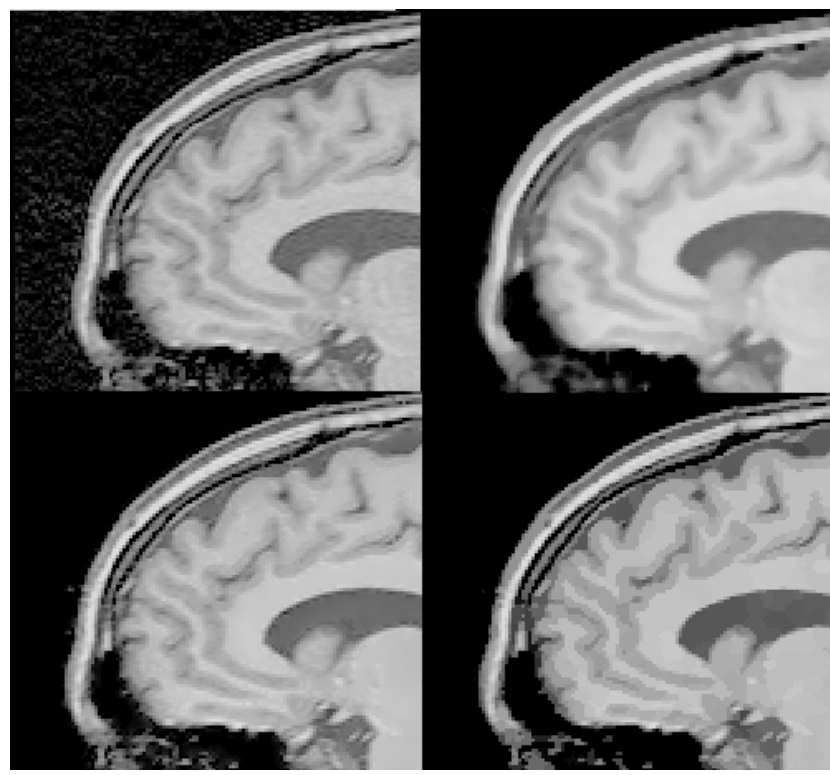

Figure 1. Restoration results. From top left to bottom right: the original slice; the median image; the anisotropic diffusion result; the proposed entropic-MRF filter

[3] D. Donoho. Nonlinear wavelet methods for recovery of signals, densities and spectra from indirect and noisy data. In Proc. Symposia Applied Mathematics, volume 47, pages 173-205, 1993.

[4] S. Geman and D. Geman. Stochastic relaxation, Gibbs distributions and the Bayesian restoration of images. IEEE Trans. PAMI, 6(6):721-741, 1984.

[5] G. Gerig, O. Kübler, R. Kikinis, and F. Jolesz. Nonlinear anisotropic filtering of MRI data. IEEE Trans. on Medical Imaging, 11(2), 1992.

[6] A. B. Hamza and H. Krim. Image denoising: A nonlinear robust statistical approach. IEEE Trans. on Signal Processing, 49(12):1-10, 2001.

[7] K. Held, E. Kops, B. Krause, W. Wells, R. Kikinis, and H.W. Gärtner. Markov random field segmentation of brain MR images. IEEE Trans. on Medical Imaging, 16(6):878-886, 1997.

[8] R. Nowak. Wavelet-based Rician noise removal for magnetic resonance imaging. IEEE Trans. on Image Processing, 8(10):1408-1419, 1999.

[9] S. Olsen. Noise variance estimation in images. In $8^{\text {th }}$ Scandinavian Conf. on Image Analysis, Norway, 1993.

[10] C. Shannon. A mathematical theory of communication. The Bell System Technical Journal, 27:379-423, 1948.

[11] C. Westin, L. Wigström, T. Loock, L. Sjöqvist, R. Kikinis, and $\mathrm{H}$. Knutsson. Three-dimensional adaptive filtering in magnetic resonance angiography. J. Magnetic Resonance Imaging, 14:63-71, 2001. 